\title{
Theoretical Evidence for Low-Spin Ground States of Early Interstitial and Late Substitutional $3 d$ Transition-Metal Ions in Silicon
}

\author{
F. Beeler, (1) O. K. Andersen, (1) and M. Scheffler ${ }^{(1),(2)}$ \\ (1) Max-Planck-Institut für Festkörperforschung, D-7000 Stuttgart, Federal Republic of Germany \\ (2) Physikalisch-Technische Bundesanstalt, D-3300 Braunschweig, Federal Republic of Germany
} (Received 20 May 1985)

Ground-state total energies and spins have been calculated for all interstitial and substitutional $3 d$ ions in crystalline $\mathrm{Si}$ by use of spin-unrestricted density-functional theory plus the linear muffintin-orbital Green's-function method. The calculated deep donor and acceptor levels reproduce for the first time all experimentally observed transitions. The early $3 d$ interstitial ions and the late $3 d$ substitutional ions are calculated to have low spin. This is in conflict with the generally accepted model due to Ludwig and Woodbury.

PACS numbers: 71.55.Fr, 61.70.Rj, 71.70.Ch

Basic studies of transition-metal (TM) impurities in semiconductors have received growing interest in recent years. $^{1-10}$ The reason is partly technological, because, by virtue of having a multitude of electronic levels deep in the gap and a strong electron-phonon coupling, these impurities are efficient centers for the radiationless recombination of electrons and holes. ${ }^{1}$ The iron-group ( $3 d$ ) TM's are interstitial diffusers in $\mathrm{Si}$ and they stay in thermal equilibrium at-or in the cases of $\mathrm{Ni}$ and $\mathrm{Cu}$, close to-the tetrahedral interstitial site. ${ }^{2}$ Substitutional $3 d$ impurities are normally formed by recombination of $3 d$ interstitials with vacancies. $^{2}$

Our qualitative understanding of the electronic structure of single $3 d$ TM impurities in $\mathrm{Si}$ is based on the pioneering work of Ludwig and Woodbury (LW). ${ }^{3}$ They developed a model to interpret and analyze the electron paramagnetic resonance (EPR) of various interstitial and substitutional TM ions. According to this model an ion with $n$ valence electrons is in a high-spin $d^{n}$ configuration if incorporated at an interstitial site and in a high-spin $d^{n-4}$ configuration if incorporated at a substitutional site. In the latter case there are only $n-4$ electrons in localized $3 d$-like states because four of the $n$ electrons supplied by the ion are supposed to substitute for the four Si electrons removed from the valence band. That the ground states have high spin follows from the assumption that Hund's rule acts as in an atom where there is no crystal field. The orbital symmetry is specified in the LW model by the assumption that, for an ion at the tetrahedral interstitial site, the crystal field places the localized $d$ states of $t_{2}$ symmetry energetically lower than the localized $d$ states of $e$ symmetry, such that the former are occupied preferentially. For substitutional ions the crystal field has the same symmetry but the opposite sign.

This model is generally accepted ${ }^{2,4}$ and it has often been applied also to such $3 d$ ions where no EPR data exist. Since the real electronic structure involves extended states as well as localized states with fairly strong Coulomb interactions, a microscopic justification of the LW model and a discussion of its validity range by means of a theoretical calculation of suffi- cient accuracy has not yet been given. This is the aim of the present Letter, which gives results of spinunrestricted, density-functional $(\mathrm{SDF})^{5}$ calculations for single TM ions incorporated in an otherwise perfect infinite Si crystal. For various early interstitial TM ions, as well as for various late substitutional TM ions, we shall find low-spin ground states and, hence, a break down of the LW model for these systems. Our calculations also predict which charge states (ions) are possible for a given TM impurity and they yield the donor and acceptor levels, that is, the energies for transitions between the ground states of adjacent charge states when an electron or hole is transferred to the conduction or valence band. ${ }^{6}$

The previous work by DeLeo, Watkins, and Fowler, ${ }^{7}$ had the same aim as ours and it discussed the relation between spin-restricted and spin-unrestricted approaches to the many-electron problem. Unfortunately, the actual calculations were hampered by large uncertainties due to the use of finite clusters to simulate the Si host. Zunger and Lindefelt ${ }^{8}$ used the proper host, but only the most recent calculations by Katayama-Yoshida and Zunger ${ }^{9,10}$ were spin polarized, in addition to including a self-interaction correction. ${ }^{11}$ The results for interstitial $\mathrm{Fe}^{9}$ are similar to ours. A more detailed discussion of and comparisons with the earlier calculations ${ }^{7-9}$ is given elsewhere. ${ }^{12}$

Our self-consistent SDF calculations were performed with the linear muffin-tin-orbital (LMTO) Green's-function method ${ }^{13}$ in the atomic-spheres, local SDF, ${ }^{5}$ and frozen-core approximations. The basis set consisted of the $s, p$, and $d$ MTO's on all Si and interstitial sites. The perturbation of the effective single-particle potential was included inside the nine atomic spheres, each of radius 2.526 bohr, containing the impurity, the four nearest $\mathrm{Si}$, and the four nearest interstitial sites. For all ions considered the net induced charge inside this region was found to differ by less than 0.05 electron from the asymptotic value $q / \epsilon$, where $q=Z-n$ is the charge of the TM ion, $Z$ the core charge, and $\epsilon(\approx 12)$ the Si dielectric constant. For charge states the contribution to the total energy of the long-range Coulomb potential, $-2 q(\epsilon r)^{-1} \mathrm{Ry}$, 
was taken to be $-q(0.1 \mathrm{eV}) .^{14}$ Relaxations of the atomic positions were not considered. In our otherwise parameter-free calculations it was found necessary to correct the local density-functional band gap of pure Si so that it equaled the experimental value of $1.12 \mathrm{eV}$. This was done by shifting all unoccupied Si bands upwards by $0.6 \mathrm{eV}$ before calculating the Green's function of the Si host. ${ }^{15}$

The SDF single-particle spectrum calculated for the ground states of the neutral impurities is sketched in Fig. 1. The spin-up and spin-down valence bands, indicated by the lower boxes, both extend from -11.9 to $0 \mathrm{eV}$ and, far from the impurity, their states equal the pure $\mathrm{Si}$ states. The number of valence-band states is as in the pure crystal, provided that the defect-induced resonances in the valence band explicitly shown in Fig. 1 are not referred to as valence-band states. This holds for interstitials as well as for substitutionals although, in the latter case, the substitution in the bonds of the four Si $s p^{3}$ orbitals by the four TM $4 s$ and $3 d\left(t_{2}\right)$ orbitals causes drastic rearrangements in the valence band. Consequently, for interstitials $n$ electrons, and for substitutional $n-4$ electrons, are left to occupy states other than valence-band states. Those are the $e$ and $t_{2}$ states labeled in the figure by dashed and full lines, respectively. Such a state is truely localized only if its energy is in the gap. Otherwise it matches onto band states whereby the discrete level broadens into a resonance. There is no extra state in the valence band or in the gap derived from the $4 s$ orbital. This is all consistent with the LW model, and so are the orderings of the $e$ and $t_{2}$ levels (for the same spin direction) which we shall now discuss.

For the interstitials the $t_{2}$ and $e$ states are, to a first approximation, nonbonding, and the reason why $t_{2}$ lies below $e$ is that, at the interstitial site, the pure $\mathrm{Si}$ valence band has strong $t_{2}$ and some $e$ character, while the lower part of the pure $\mathrm{Si}$ conduction band has strong $t_{2}$ but practically no $e$ character. The interaction between these band states and the impurity $t_{2}$ and $e$ orbitals then, essentially, confines the energy of the $t_{2}$ state to the gap and lifts the energy of the $e$ state. Consistently herewith we find that the less-interacting $e$ state is the more localized, whereas the probability, $R_{0}\left(t_{2}\right)$, that a $t_{2}$ electron is inside the atomic sphere at the impurity is only 0.30 in $\mathrm{Ti}^{0}$ and increases to 0.50 in $\mathrm{Co}^{0}$; the $e$ probability is approximately 0.55 in all neutral interstitials. The $e-t_{2}$ splitting is therefore a ligand-field-rather than a crystal-field-effect. Going through the series, the $e$ and, in particular, the $t_{2}$ levels move down more slowly than the $3 d$-orbital energy, which falls by $3.5 \mathrm{eV}$ between $\mathrm{Ti}^{0}$ and $\mathrm{Ni}^{0}$. The $e-t_{2}$ splitting decreases from $1.5 \mathrm{eV}$ in $\mathrm{Ti}^{0}$ to $0.4 \mathrm{eV}$ in $\mathrm{Ni}^{0}$.

For the substitutionals the $e$ states lie below the $t_{2}$ states because the $e$ states are nonbonding whereas the $t_{2}$ states are essentially antibonding partners of the impurity-host bonds. The $e$ states are thus well localized and the $t_{2}$ states are nearly delocalized; $R_{0}(e)=0.66$ and $R_{0}\left(t_{2}\right)$ decreases from 0.30 in $\mathrm{Cr}^{0}$ to 0.14 in $\mathrm{Cu}^{0}$. The $t_{2}$-e splitting increases from $0.5 \mathrm{eV}$ in $\mathrm{Ti}^{0}$ to $1.2 \mathrm{eV}$ in $\mathrm{Ni}^{0}$.

In Fig. 1 interstitial $\mathrm{Ti}^{0}$ and $\mathrm{V}^{0}$ and substitutional $\mathrm{Fe}^{0}, \mathrm{Co}^{0}$, and $\mathrm{Ni}^{0}$ have low spin. We shall now see that it is the covalency found for these early interstitials and late substitutionals which prevent them from having high-spin ground states. For interstitial $\mathrm{V}^{0}$, for instance, the high-spin state would have the two $t_{2}$ electrons promoted to the $e^{\dagger}$ state and thus have $2 S=5$ rather than 1. The promotion energy of $0.95 \mathrm{eV}$ is twice the $e^{\dagger}-t_{2}{ }_{1}$ difference minus the electronic relaxation energy or, approximately, twice the $e^{\uparrow}-t_{2}{ }^{1}$ difference calculated for a transition state in which there is one electron in the $t_{2} \downarrow$ state and one in the $e^{\uparrow}$ state. The ground state thus has low spin if the ligand-field splitting exceeds the spin splitting calculated for the transition state. The spin splitting is, in first-order perturbation theory, $m_{0} I_{0} R_{0}=2 S I_{0}\left(m_{0} / m\right) R_{0}$. Here, $m_{0}$ and $m=2 S$ are the integrals of the spin density in the impurity sphere and in all space, respectively. $I_{0}$ is the TM Stoner parameter. By fitting the above expression to our calculated spin splittings we obtain $I_{0}=0.9 \pm 0.1 \mathrm{eV}$ for all ions and spin states considered. This agrees well with values calculated ${ }^{16}$ for the elemental TM's when taking the compression to a Wigner-Seitz radius of 2.526 into account. The result of this analysis is therefore that covalency not only causes large ligand fields but also considerably reduces the effective exchange constants for the TM impurities by localization factors for the gap state $\left(R_{0}\right)$ and for the spin density $\left(m_{0} / m\right)$. The latter factors are given for the ground states in Table I.

In Table I we list all the charge states (ions) which we found to exist and, for each of these, the electronic configuration which gave the lowest SDF total energy. The corresponding many-electron ground state is specified by its spin multiplicity $(2 S+1)$ and orbital $(L)$ in the tetrahedral point group. Low-spin ground states were found for the interstitials $\mathrm{Ti}^{0}, \mathrm{~V}^{+}, \mathrm{Ti}^{-}, \mathrm{V}^{0}$, $\mathrm{Cr}^{+}, \mathrm{V}^{-}$, and $\mathrm{Cr}^{0}$, as well as for the substitutionals $\mathrm{Fe}^{0}, \mathrm{Co}^{+}, \mathrm{Ni}^{+}+, \mathrm{Co}^{0}, \mathrm{Ni}^{+}, \mathrm{Co}^{-}, \mathrm{Ni}^{0}$, and $\mathrm{Cu}^{+}$. The ions for which EPR data exist ${ }^{2-4}$ are underlined in the table. These ions all have high spin in agreement with the LW model and in agreement with our calculations, except for interstitial $\mathrm{Cr}^{+}$and $\mathrm{Cr}^{0}$. Recent electronnuclear double-resonance spectra ${ }^{17}$ for interstitial $\mathrm{Fe}^{0}$ are in even better agreement with our $m_{0} / m$ value given in the table than with the previous calculation. ${ }^{9}$ In the future, it should be possible to obtain further experimental checks of our spin-density values.

Established experimental acceptor $(-/ 0)$, donior $(0 /+)$, and double donor $(+/++)$ levels exist ${ }^{2,6}$ for interstitial $\mathrm{Ti}, \mathrm{V}, \mathrm{Cr}, \mathrm{Mn}$, and $\mathrm{Fe}$, and they are shown in Fig. 2 by the dotted lines. The solid lines are our 


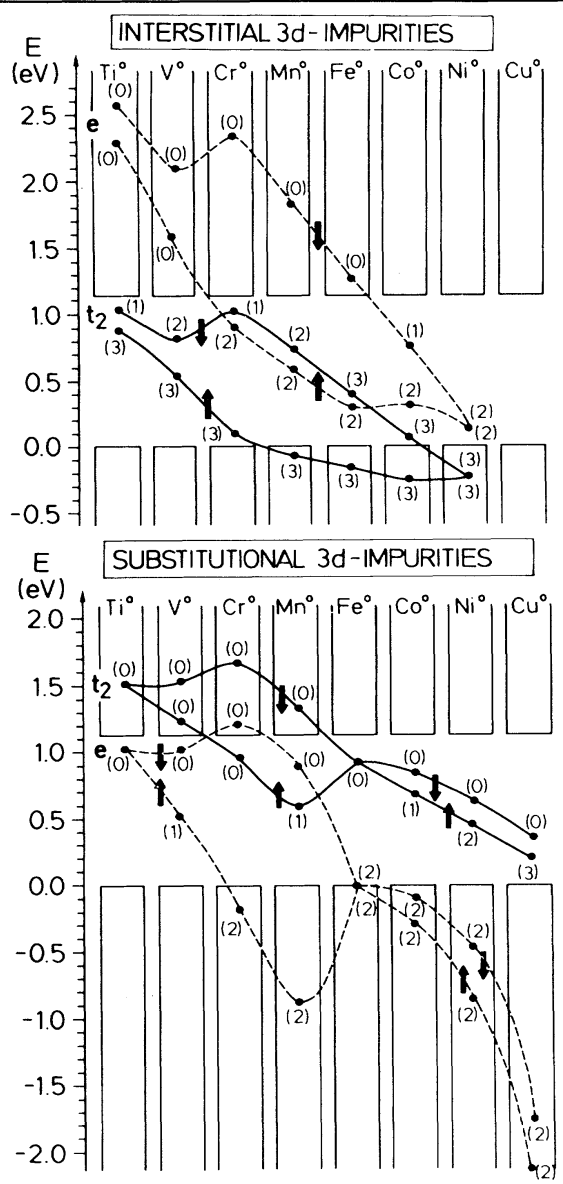

FIG. 1. Single-particle energies calculated for the ground states (Table I) of the neutral impurities. A number in parentheses gives the occupancy of a localized gap state, or of a resonance in the valence or conduction band. For interstitial $\mathrm{Cr}^{0}$ the high-spin state was used. Interstitial $\mathrm{Cu}$ only exists in the single-positive charge state.

calculated transition energies. For $\mathrm{Cr}^{0}$ and $\mathrm{Cr}^{+}$we used the experimental, high-spin ground states given in parentheses in Table I. The good agreement then

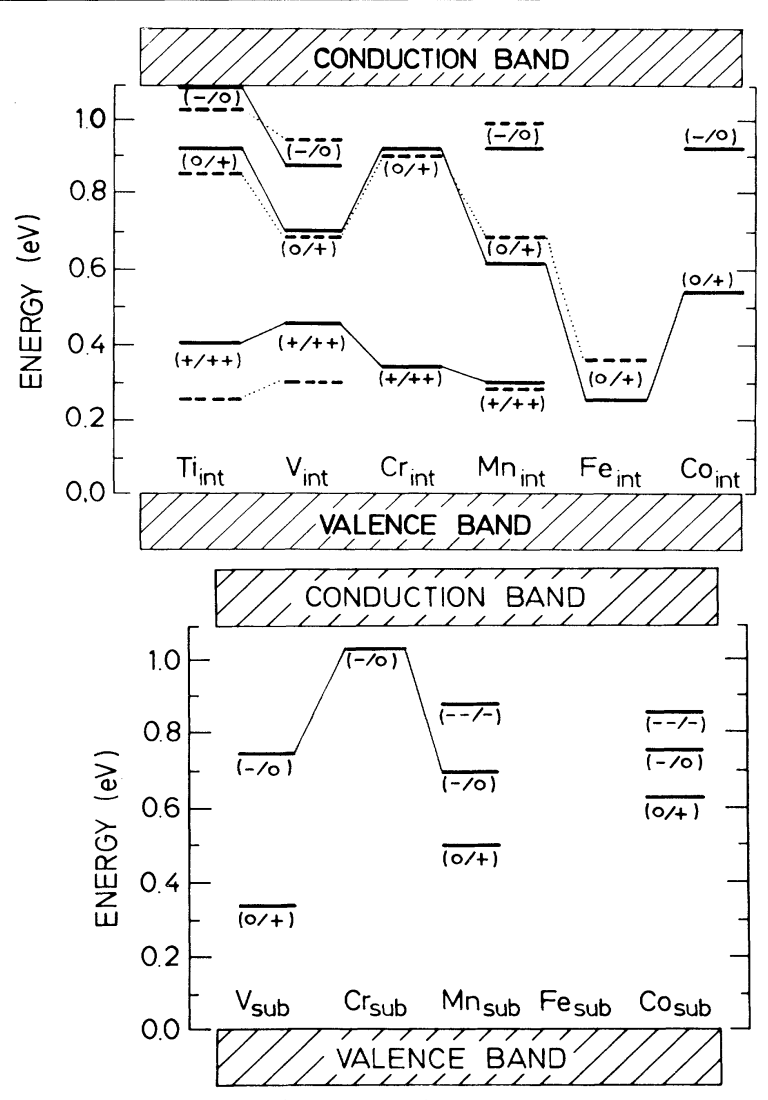

FIG. 2. Calculated (solid lines) and experimental (dotted lines) (Refs. 2 and 6) acceptor and donor levels for interstitial and substitutional impurities in $\mathrm{Si}$. The calculated ground states are given in Table I, but for interstitial $\mathrm{Cr}^{0}$ and $\mathrm{Cr}^{+}$we used the high-spin states. For substitutional Ti and Fe we found no deep levels.

obtained between theory and experiment supports our prediction that $\mathrm{Ti}$ and $\mathrm{V}$ have low-spin ground states. To see this in more detail we now relate the donor and acceptor levels in Fig. 2 to the SDF single-particle energies in Fig. 1: As seen in Table I, the Ti $(-/ 0)$ and $(0 /+)$ transitions involve a change of the occupation

TABLE I. Calculated ground states. For interstitial $\mathrm{Cr}^{0}$ and $\mathrm{Cr}^{+}$the states given in parentheses have higher total energies than the calculated ground states by 0.7 and 0.2 $\mathrm{eV}$, respectively. EPR-identified states are underlined.

\begin{tabular}{|c|c|c|c|c|c|c|c|c|c|c|c|c|c|c|c|}
\hline & $d^{n}$ & $d^{2}$ & $d^{3}$ & $d^{d}$ & & & $d^{5}$ & & $d^{6}$ & & $d^{7}$ & $d^{8}$ & 8 & $d^{9}$ & $d^{10}$ \\
\hline & $\begin{array}{c}T M^{q} \\
e \\
t_{2} \\
2 S+1 L \\
m_{0} / m(\%)\end{array}$ & $\begin{array}{c}\mathrm{Ti}^{++} \\
11 \\
{ }^{3} \mathrm{~T} \\
48\end{array}$ & $\begin{array}{l}T^{T+} \underline{V}^{++} \\
19 \dagger \\
4{ }^{4} \mathrm{~A}_{2} \\
42 \quad 55\end{array}$ & 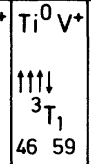 & $\begin{array}{l}\mathrm{Cr}^{++} \\
1 \\
114 \\
5 \mathrm{E} \\
70\end{array}$ & 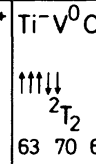 & 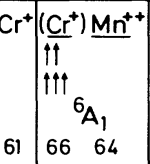 & 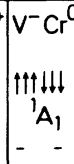 & 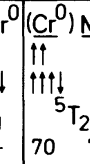 & & $\begin{array}{l}\mathrm{Mn}^{0}{ }^{0} \mathrm{Fe}^{+} \\
t \uparrow 11 ! \\
{ }^{4} \mathrm{~T}_{1} \\
81 \quad 73\end{array}$ & 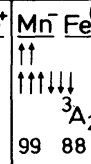 & $\begin{array}{ll}2 & \\
3 & 73\end{array}$ & $\begin{array}{c}C_{0}{ }^{0} \\
1 \uparrow \downarrow \\
\uparrow \dagger \uparrow \downarrow \downarrow \downarrow \\
2 \mathrm{E} \\
84\end{array}$ & $\begin{array}{l}\mathrm{Co}^{-} \mathrm{Ni}^{0} \mathrm{Cu}^{+} \\
\uparrow \uparrow \downarrow \downarrow \\
\uparrow \uparrow \uparrow \downarrow \downarrow \downarrow \\
{ }^{1} \mathrm{~A}_{1} \\
--\end{array}$ \\
\hline & $d^{n-4}$ & $d^{0}$ & $d^{\prime}$ & $d^{2}$ & & $d^{3}$ & $d^{4}$ & & $d$ & & & $d^{6}$ & & $d^{7}$ & \\
\hline & $\begin{array}{c}M^{q} \\
t_{2} \\
e \\
2 S+1 L \\
m_{0} / m(\%)\end{array}$ & ${ }^{1} A_{1}$ & \begin{tabular}{l|l}
$v^{+}$ & $v^{0}$ \\
$\vdots$ \\
2 \\
${ }^{2} E$ \\
99
\end{tabular} & 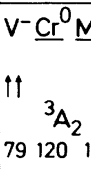 & & $\begin{array}{cc}\mathrm{Cr}^{-} \mathrm{Mn}^{0} \\
{ }^{4} \mathrm{~T}_{1} \\
{ }^{4} \mathrm{~T}_{1} \\
94 & 100\end{array}$ & 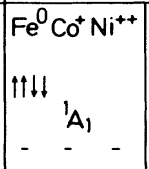 & $\mid \begin{array}{c}\mathrm{Mn}^{-} \\
11 \\
11 \\
5 \mathrm{~T}_{2} \\
81\end{array}$ & 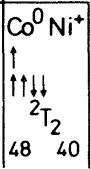 & 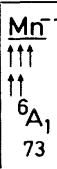 & $\mid \begin{array}{l}C^{-1} \\
11 \\
11+1 \\
61\end{array}$ & $\begin{array}{ll}{ }^{3} \mathrm{~T}_{1} & \\
38 \quad 22\end{array}$ & $\begin{array}{l}C^{--} \\
11 \uparrow \\
\uparrow \uparrow 14 \\
\\
60\end{array}$ & $\mathrm{Ni}^{-} \mathrm{Cu}$ & $u^{0}$ \\
\hline
\end{tabular}


of the $t_{2}$ state. Therefore, in the absence of electronic relaxations $(U=0)$, these levels would coincide with the $t_{2} \downarrow$ energy in Fig. 1. Similarly, the three $V$ levels, the $(0 /+)$ level of $\mathrm{Cr}$, the three Mn levels, and the $\mathrm{Fe}$ $(0 /+)$ level all relate to the $t_{2}{ }^{1}$ energy. The characteristic jump between $\mathrm{V}$ and $\mathrm{Cr}$, interrupting the otherwise monotonous decrease of these levels through the series, thus reflects the behavior of the $t_{2}$ energy in Fig. 1 and is caused by the switch from low-to high-spin ground states. Had the $\mathrm{V}$ and $\mathrm{Ti}$ ions not been in lowspin, but in high-spin states, then the $\mathrm{V}(-/ 0)$ level would still be assigned to $t_{2} \downarrow$ but the $\mathrm{V}(0 /+)$ and $(+/++)$ levels, as well as the $\mathrm{Ti}(-/ 0)$ and $(0 /+)$ levels, should be assigned to $e^{\uparrow}$. In that case general agreement with experimental donor and acceptor levels cannot be achieved.

The experimental facts that interstitial $\mathrm{Cr}$ and $\mathrm{Fe}$ have no acceptor level in the gap are successfully reproduced by our calculation which shows that $\mathrm{Cr}^{-}$ and $\mathrm{Fe}^{-}$are unstable because, for the former, the $t_{2} \downarrow$ and, for the latter, the $e^{\downarrow}$ level is in the conduction band. ${ }^{18}$ The $\mathrm{Cr}$ double-donor level obtained by us and assigned to $e^{\uparrow}$ is not found experimentally. ${ }^{19}$ This failure of our $\mathrm{Cr}^{++}$calculation is consistent with the above-mentioned failures for $\mathrm{Cr}^{0}$ and $\mathrm{Cr}^{+}$in the sense that a general lowering of the calculated $e^{\dagger}$ level by about $0.35 \mathrm{eV}$ would bring all theoretical ground states and level positions in agreement with the experimental data. This error we attribute to the local SDF and the gap correction, ${ }^{15}$ to the atomic-spheres approximation, and to defect-induced lattice distortions (symmetric and nonsymmetric) neglected in our calculations. In particular, we believe that for the early, relatively large TM ions there might be an outwards relaxation of the $\mathrm{Si}$ neighbors in the high-spin states, but not in the low-spin states. Such a magnetic-pressure effect ${ }^{16}$ would tend to stabilize the high-spin states.

There are no established experimental-level positions for TM impurities at the substitutional site. The recent finding ${ }^{20}$ that for $\mathrm{Mn}$ there is a $(0 /+)$ level at $0.38 \mathrm{eV}$, and no levels below, may be an exception. It agrees well with a result of our calculations shown in the bottom half of Fig. 2. For the substitutionals the high- to low-spin transition occurs to the right of $\mathrm{Mn}$.

We gratefully acknowledge help from O. Jepsen, O. Gunnarsson, H. J. Queisser, P. Vogl, G. D. Watkins, and E. Weber.

${ }^{1}$ J. Dietl, D. Helmreich, and E. Sirtl, Cryst. Growth Prop. Appl. 5, 43 (1981).

${ }^{2}$ E. R. Weber, Appl. Phys. A 30, 1 (1983).

${ }^{3}$ G. W. Ludwig and H. H. Woodbury, Solid State Phys. 13, 223 (1962).

${ }^{4}$ D. A. von Wezep and C. A. J. Ammerlaan, J. Electron. Mater. 14, 863 (1985).
${ }^{5}$ U. von Barth and L. Hedin, J. Phys. C 5, 1629 (1972). Use of another local SDF changes our single-particle energies by less than $0.1 \mathrm{eV}$.

${ }^{6} \mathrm{~K}$. Graff and H. Pieper, in Semiconductor Silicon 81-5 (Electrochemical Society, Pennington, N.J., 1981), p. 331; L. C. Kimerling, J. L. Benton, and J. J. Rubin, in Defects and Radiation Effects in Semiconductors (Institute of Physics, London, 1981), p. 217.

${ }^{7}$ G. G. DeLeo, G. D. Watkins, and W. B. Fowler, Phys. Rev. B 23, 1851 (1981), and 25, 4962,4972 (1982).

${ }^{8}$ A. Zunger and U. Lindefelt, Phys. Rev. B 26, 5989 (1982), and 27, 1191 (1983), and 28, 3628 (1983).

${ }^{9}$ H. Katayama-Yoshida and A. Zunger, Phys. Rev. Lett. 53, 1256 (1984), and Phys. Rev. B 31, 7877 (1985).

${ }^{10}$ SDF-SIC calculations for the interstitial $3 d$ impurities by H. Katayama-Yoshida and A. Zunger were presented together with ours in Bull. Am. Phys. Soc. 30, 302 (1985). The results and conclusions are very similar but there are differences in detail. After submission of the present manuscript a report of the SDF-SIC calculations appeared. [Phys. Rev. B 31, 8317 (1985).]

${ }^{11}$ We did not use the SIC because it gave no improvement over local SDF calculations of total-energy differences between various valence-electron configurations for $3 d$ atoms. See O. Gunnarsson and R. O. Jones, Solid State Commun. 37, 249 (1981).

${ }^{12}$ F. Beeler, O. K. Andersen, and M. Scheffler, in Proceedings of the Materials Research Society Meeting, San Francisco, 1985 (to be published).

${ }^{13}$ O. Gunnarsson, O. Jepsen, and O. K. Andersen, Phys. Rev. B 27, 7144 (1983).

${ }^{14}$ C. O. Rodriguez, S. Brand, and M. Jaros, J. Phys. C 13, L333 (1980).

${ }^{15}$ The application of this scissor operator [G. Baraff and M. Schlüter, Phys. Rev. B 30, 3460 (1984)] leaves the total energy of the host unchanged but could lead to a total energy of the perturbed system which is too high by roughly 0.6 $\mathrm{eV}$ times the number of occupied, conduction-band-derived states. This unphysical side effect is reduced in the LMTO Green's-function formalism [Ref. 13, and O. K. Andersen, O. Jepsen, and D. Glötzel, in Proceedings of the Enrico Fermi International School of Physics, Course LXXXIX, edited by F. Bassani, F. Fumi, and M. P. Tosi (North-Holland, Amsterdam, 1985)] because here the scissor operator, $\left\langle\chi_{i}^{0}|\hat{S}| \chi_{j}^{0}\right\rangle$ in the orthogonal MTO representation ( $i$ labels the site and angular momentum of the orbital), for the pure host transforms into

$$
\left(\Delta_{i} / \Delta_{i}^{0}\right)^{1 / 2}\left\langle\chi_{i}^{0}|\hat{S}| \chi_{j}^{0}\right\rangle\left(\Delta_{j} / \Delta_{j}^{0}\right)^{1 / 2} \approx\left\langle\chi_{i}|\hat{S}| \chi_{j}\right\rangle,
$$

for the perturbed system. $\Delta$ is the potential parameter describing the squared amplitude of the MTO tail and, for a TM $d$ orbital, it is from $4(\mathrm{Ti})$ to $20(\mathrm{Cu})$ times smaller than $\Delta^{0}$ for the substituted Si or interstitial-sphere $d$ orbital.

${ }^{16}$ Andersen, Jepsen, and Glötzel, Ref. 15.

${ }^{17}$ S. Greulich-Weber, J. R. Niklas, E. R. Weber, and J.-M. Spaeth, Phys. Rev. B 30, 6292 (1984).

${ }^{18}$ For the case of interstitial $\mathrm{Fe}$ this was also found previously (Ref. 9).

${ }^{19}$ H. Conzelmann, K. Graff, and E. R. Weber, Appl. Phys. A 30, 169 (1983).

${ }^{20}$ R. Czaputa, H. Feichtinger, J. Oswald, M. Haider, and H. Sitter, Phys. Rev. Lett. 55, 758 (1985). 